\title{
Relieving in-patient boredom in general hospitals: the evidence for intervention and practical ideas
}

\author{
Rachel Steele \& Keith Linsley
}

\begin{abstract}
SUMMARY
Boredom is highly prevalent among general hospital in-patients. Self-help strategies suit some patients, but for others hospitals need to stimulate opportunities for them to experience meaningful relationships and roles and a sense of control. In-patients' well-being and levels of boredom may be affected by no-smoking policies, hospital design, access to natural light, nature scenes and indoor or outdoor gardens. Alleviating boredom in elderly patients with reduced cognitive function may be particularly challenging. Healthcare professionals may face the wider challenge of fully engaging with patients' psychosocial needs, given the biomedical model that privileges the 'traditional medical history' over the more holistic communication model. Engaging with patients' psychosocial needs is consistent with managing their experience of 'sickness' rather than focusing on the narrower concept of 'disease'.
\end{abstract}

\section{LEARNING OBJECTIVES}

- Appreciate how facilitating in-patients' opportunities to experience meaningful relationships, roles and a sense of control is important in alleviating boredom

- Articulate how smoking cessation may affect levels of boredom, given the effect smoking has on key neurotransmitters

- Understand how alleviating boredom may be challenging in particular in-patient populations

\section{DECLARATION OF INTEREST}

None

Boredom is a significant problem on psychiatric in-patient wards (National Institute for Health and Clinical Excellence 2011a; Royal College of Psychiatrists 2011), as we discussed in a previous article in Advances (Steele 2013). So why are we revisiting the topic for patients in general hospitals? First, because there are fundamental differences between the experience of being an in-patient in a general hospital and being an inpatient in a psychiatric unit: physical health conditions do not carry the same stigma as mental illnesses and typically when people experience physical illness, social resources are mobilised to support them (Byrne 2000). And second, because boredom among acute hospital in-patients significantly affects clinical outcomes. This should be of concern to all health professionals, including psychiatrists who provide liaison support in general hospitals.

\section{Overview of the evidence}

\section{Prevalence of boredom in general hospitals}

A number of studies have examined the nature of 'boredom' in patients in a general hospital setting and all report that it is common and that it has a secondary impact on the patient's health. For example, Hyland (1996) reported that 86\% of inpatients with cancer indicated that they had 'time on their hands' and 50\% said that they were bored. Likewise, Hardy $\&$ West (1994) found that $70 \%$ of patients they surveyed had 'not enough to do in hospital'. An observational study of activity found that patients were passive for $87 \%$ of the time (Nolan 1995). The British Medical Association (BMA) has recommended that measures should be put in place to allow hospital in-patients to participate in recreational and creative therapies (BMA Science \& Education 2011).

\section{Preventing boredom}

Naturally, coming into hospital brings many changes and disruptions to an individual's life. For a short stay, this may have little bearing on the patient's well-being or treatment outcome and, in some cases, boredom can be eased by the individual or their family and friends by employing relatively straightforward self-help strategies (Box 1). But as the length or complexity of admission progresses, it becomes more important for staff to be aware of the presence of boredom in patients. Indeed, perhaps the most important factor in recognition is to be aware that it is highly likely to be present. The second important step is for hospital staff to feel comfortable enough to ask the patient.
Rachel Steele is a clinical librarian with Tees, Esk and Wear Valleys NHS Foundation Trust. Her main interests include the role of the clinical librarian/informationist in facilitating access to the evidence base in all aspects of mental health services, as well as educational/ CPD interventions to develop health professionals' skills in using evidence in their practice. She has recently completed an MSc in Health Services Research at the University of York. Keith Linsley is a consultant psychiatrist with a special interest in liaison psychiatry. He is based at Chester-le-Street Health Centre (Tees, Esk and Wear Valleys NHS Foundation Trust) and the University Hospital of North Durham (County Durham and Darlington NHS Foundation Trust). Correspondence Rachel Steele, Library and Information Service, Education Centre, Lanchester Road Hospital, Durham DH1 5RD, UK Email: rachelsteele@nhs.net 
BOX 1 Self-help strategies for alleviating boredom

Several websites offer handy tips on managing someone's stay in hospital. There are excellent examples of good practice: Roswell Park Cancer Institute, for example, offers the following advice. Although this is an unusually well-equipped centre, other hospitals can aspire to replicate its practices:

- stay connected through the Institute's free wi-fi access and laptop rentals

- watch TV or rent films from the Institute in your room

- ask visiting friends and family to bring in activities that you normally enjoy (such as reading material or board games)

- participate in something new or ask nurses or occupational therapists to set up an activity with other patients of the same age or diagnosis

- exercise, if your health permits www.roswellpark.org/cancertalk/201303/how-beatboredom-during-long-hospital-stays

In this article we highlight important theoretical and conceptual themes that inform understanding of the holistic nature of boredom among acute hospital in-patients. We then discuss some of the interventions studied for addressing boredom and reducing its potential secondary impact on health and healthcare resource use. Our aim is not necessarily to persuade units to directly replicate the examples we highlight, but rather to stimulate thought within general hospitals and the mental health teams that support them. We particularly wish to emphasise practical measures that are neither costly nor time-consuming and would not require particular training.

\section{Effects of boredom on clinical outcomes}

There is evidence, albeit limited, that attests to the importance of attending to patients' psychosocial needs in influencing length of stay in general hospitals. For example, in a Canadian study the severity of psychosocial problems was found to be significant in explaining the difference between actual and target length of stay for acute care hospital patients referred to Social Services (Lechman 2006). In a second study by the Canadian researchers, the severity of psychosocial problems of psychiatric and medical/surgical patients was reported to be more significant in predicting length of stay than was severity of medical condition (Keefler 2001). The need to consider preoperative psychosocial factors as predictors of length of stay following heart transplantation has also been highlighted (Grady 1999).

\section{Hope and boredom}

Hope 'has been shown to be important among cancer patients in coping, perceived control over the illness, and psychologic adjustment' (Vellone 2006). Interestingly, hope was found to be negatively correlated with boredom during hospital stays of Italian patients with cancer (Vellone 2006). Another study found that stimulation and avoidance of boredom were predictive of quality of life among advanced cancer patients (Inman 2003). Given that hope is highly important for promoting treatment effectiveness, its correlation with boredom is significant and suggests that addressing boredom could have much wider implications for patients with cancer in terms of experiencing hope and coping with their illness in multiple ways.

\section{Conditions that may contribute to or falsely present as boredom}

Although not a focus of this article, readers should be mindful of other conditions that may be contributing to boredom or be falsely presenting as boredom (or lack of drive) (Box 2). Particular problems can arise in patients with established and recognised mental disorders such as schizophrenia or those with intellectual disabilities (often referred to as learning disabilities in UK healthcare services). We would recommend in this case first asking the patient whether they have noticed a change in themselves and second, seeking corroboration and information about their usual behaviour, volition and functioning from someone who knows them well. It is also worth considering that some treatments that patients receive in general hospitals can affect volition.

\section{The patient passport}

An instructive example of a practical approach that can be used with patients who have preexisting mental disorders is the creation of a one-page patient passport. Patient passports were designed to provide individualised information about people with intellectual disabilities to enable healthcare professionals to better understand

BOX 2 Conditions that may mimic, contribute to or falsely present as boredom

- Depression

- Frontal lobe dementia

- Korsakoff dysmnesia

- Intellectual disability

- Long-standing mental disorders such as schizophrenia 
their patients. Over their lifetime, people with intellectual disabilities are more likely to use healthcare services than is the general population, but many have negative experiences in hospitals because clinical staff struggle to understand their needs (Brodrick 2011). The passport is designed to overcome barriers to their effective treatment by highlighting concisely what the patient is like and how they might best be treated and helped in an acute hospital. A similar approach could be used for people with pre-existing psychiatric conditions when they are admitted to general hospitals.

\section{Theories of boredom}

There are two major (but not mutually exclusive) theories of boredom - situation-dependent boredom and situation-independent boredom. Situation-dependent boredom is externally driven, primarily due to a monotonous environment. In contrast, situation-independent boredom arises from internal factors (Todman 2003; Steele 2013). Proponents of situation-independent boredom believe that boredom results from an inability to use coping mechanisms such as fantasy and anticipation to escape from monotony (Todman 2003).

\section{Boredom and the experience of meaning in life}

A key internally driven sociological theory of boredom among psychiatric in-patients is that boredom is an emotion inherently linked to the experience of meaning in life, and that the three interrelated factors vital to experiencing meaning are being in meaningful relationships, having meaningful roles and having a sense of control (Binnema 2004). Although this theory is one of several competing theories of boredom, and is focused on understanding psychiatric in-patients, it offers a useful framework for understanding boredom among general hospital in-patients.

\section{Meaningful relationships while in hospital}

The diversity of demographic characteristics and physical health factors among general hospital in-patients mean that interventions designed to address their needs for meaningful relationships while in hospital must be adapted accordingly.

With children and young people, being admitted to hospital has significant implications for their continued educational and social connections (Nisselle 2012). The Royal Children's Hospital, Melbourne offers an excellent example of using the latest technologies (such as laptops and tablets) as part of a multidisciplinary educational programme to provide access to flexible learning and socialising while in hospital (Nisselle 2012).
Having the opportunity to experience meaningful relationships is also important for adult inpatients. This has clearly been demonstrated among psychiatric in-patients, who have expressed a desire for social support from peers and fellow patients (Thomas 2002) and healthcare professionals (Higgins 1999).

For older in-patients, the provision of organised meeting times/patients' clubs to promote social interaction may be helpful, given the prevalence of loneliness among older people in general (Routasalo 2009). Older people undergoing a period of rehabilitation found that opportunities for social interaction were restricted (by both physical factors and lack of staff time) (Clissett 2001). They thought that the patients' club they could attend was beneficial overall, but it appeared only to promote activity and not to address their need for social interaction. Thus, while activities may be useful in terms of filling time and providing diversionary outlets, greater thought may be required to engagement in deeper social interaction. For example, a 2-week exposure to an enriched environment, compared with a standard stroke rehabilitation ward, resulted in stroke survivors experiencing increased social interaction, increased feelings of personal control and an alleviation of boredom (Bartley 2012). Given that boredom has also been hypothesised to be related to a feeling of loss of control over one's situation, these findings are noteworthy.

\section{Loneliness and social isolation}

'Loneliness' and 'social isolation' are often assumed to be the same, but they are distinct phenomena. Social isolation relates to the frequency of an individual's contacts and to their integration into their social environment; loneliness is determined not by the frequency of contacts, but by how satisfying these social interactions are to the individual (Routasalo 2009). This theoretical distinction has implications for general hospital in-patients. Although being in hospital will not, in itself, alter a patient's level of social isolation in the community (because family, friends, acquaintances and social networks outside the hospital will remain intact), the quality of an in-patient's social interactions with visitors may result in feelings of loneliness, for example if visits are rushed because of hospital visiting hours. Furthermore, if members of the patient's social network outside the hospital do not visit at all, patients may perceive themselves to be socially isolated. Hospital staff should therefore facilitate opportunities for patients to interact with their existing social network whenever possible. 


\section{Experiencing a sense of control and having meaningful roles}

The importance to patients of experiencing a sense of control by having the opportunity to engage in autonomous activities as opposed to generic organised activities has been highlighted with reference to psychiatric in-patients (Newell 2012).

Autonomously driven activities to provide 'structured meaningful activity for elderly patients in a hospital setting' were successfully promoted by a programme in which in-patients received a purposeful visit from volunteers trained by a recreational therapist (Cumbler 2011). Women admitted to a high-risk antepartum unit during pregnancy likewise highlighted that the loss of control was a stressor for them, although they were equally keen on the provision of organised activities to relieve boredom (Richter 2007).

Indeed, organised activities may help general hospital in-patients experience meaningful roles. For example, a person-centred arts programme for hospital-based stroke patients reported that the most frequently mentioned benefits of participation included experiences of 'engagement in purposeful occupation and relief from boredom, and reconnection with valued aspects of the self' (Baumann 2013). The link between the chance to engage in meaningful roles and occupations and relief from boredom is noteworthy, as is the fact that such 'experiences of participation contrast strongly with the acute and chronic distress associated with stroke, impairment, and spending long periods of time in hospital' (Baumann 2013).

\section{Effect of smoking regulations on boredom}

Smoking was banned in England in nearly all enclosed workplaces and public spaces in 2007. Consequently, National Health Service (NHS) acute hospitals in England operate no-smoking policies on hospital grounds; similar policies are in operation in Scotland, Wales and Northern Ireland. The health benefits of this are clear, particularly considering the harmful effects of second-hand smoke on cardiovascular health (Bauld 2011). However, the potential implications of smoking bans on levels of boredom of general hospital in-patients has been underexplored in the literature.

Nicotine crosses the blood-brain barrier and binds to nicotinic cholinergic receptors, causing the release of the neurotransmitters noradrenaline, serotonin, dopamine, acetylcholine, $\gamma$-aminobutyric acid and glutamate (Campion 2008). Smoking also inhibits the enzyme monoamine oxidase $\mathrm{B}$ (MAO-B), causing dopamine levels to increase
(Campion 2008). The mesolimbic dopamine pathway is central to motivation, pleasure and reward (Wise 2004; Stahl 2008) and is also implicated in creativity (Flaherty 2011). Studies of dopamine antagonist antipsychotics have reported that some can induce negative symptoms of schizophrenia in healthy volunteers and consequently that these dopamine-blocking agents could contribute to a lack of drive and therefore to boredom (Artaloytia 2006; Park 2012). Consequently, we might hypothesise that stopping smoking suddenly may contribute to experiences of boredom, given the decrease in dopamine levels that the smoker will experience. However, there may be some differences between general hospital inpatients and psychiatric in-patients in this area. Nevertheless, the decrease in dopamine levels that will accompany sudden smoking cessation should be considered as a potential contributor to boredom among general hospital in-patient who are regular smokers.

It should be noted, however, that the widely held assumption that smoking is associated with mental health benefits was challenged by a recent systematic review (Taylor 2014). This reported that in both the general population and populations with physical or psychiatric disorders, smoking cessation was associated with reductions in anxiety, depression, mixed anxiety and depression, and stress.

\section{Boredom and cognition in elderly people}

In a study involving community-dwelling older adults in Ireland, Conroy et al (2010) found that both loneliness and proneness to boredom were associated with reduced cognitive function in older age. Although the cross-sectional nature of this investigation precludes conclusive attribution of causality, they theorise that loneliness and boredom-proneness "may have a common underlying mechanism in the failure to select and maintain attention on particular features of the social environment (loneliness) or the nonsocial environment (boredom-proneness)'. They suggest that boredom-prone individuals may lead cognitively unstimulating lives.

As mentioned above, those who espouse the theory of situation-dependent boredom argue that boredom is primarily due to a monotonous environment. They suggest that individual differences reflect a differing level of vulnerability to the absence of environmental stimuli, with individuals more prone to boredom using particular (usually maladaptive) behaviours or traits (Steele 2013). An inability to maintain attention on the non-social environment, and a 
lack of cognitive stimulation in one's life, could be regarded as a 'maladapative' trait (although a lack of cognitive stimulation in one's life is not necessarily something for which we can blame the individual). The theory of situation-independent boredom is also relevant to Conroy et al's (2010) theory on boredom-proneness because having reduced cognitive function may lead to difficulties in using escape mechanisms such as anticipation and fantasy (Todman 2003).

For the reasons put forward by Conroy et al, it is therefore likely to be challenging for staff and liaison mental health teams in general hospitals to engage patients with reduced cognitive function. Perhaps the strategy most likely to be successful with this group would be to provide structured, organised activities to ensure that the external environment is as stimulating as possible. This is probably a sensible approach given the difficulty of encouraging patients with cognitive decline to try to alleviate their own boredom by using the cognitive coping mechanisms needed to compensate for 'boring' external environments.

\section{The physical environment of the hospital}

A BMA report argues that "poor design works against the wellbeing of patients' and that hospitals must be mindful that physical surroundings can be 'psychologically supportive' (BMA Science $\&$ Education 2011: p. 11). Although the report acknowledges that financially challenged hospitals may not easily be able to make alterations to existing structures, it states that the design of new hospitals should avoid overcrowding of patients and should 'provide a variety of spaces such as a big day room, a dining room that is well lit and ventilated and a spacious lobby and corridors and give sufficient attention to natural and artificial lighting' (p. 11). Noting that the structural environment of hospitals can significantly affect in-patients' social interaction, the report makes practical suggestions that hospital staff can implement, such as ensuring that room layouts make it easy for patients to interact with one another and with friends and family, and providing lounges with comfortable furniture that people can arrange to suit themselves.

\section{Natural light}

The idea that natural light plays an important role in promoting recovery and improving well-being is intuitively appealing. Indeed, a retrospective comparison of patients in a cardiac intensive care unit (ICU) after myocardial infarction reported that the length of stay for women was less in sunny rooms (2.3 days) than in dull rooms (3.3 days), although there was no significant difference for men (Beauchemin 1998). At a practical level, it may be difficult to ensure that all patients have access to sunny rooms, given pressures on hospital beds and facilities. However, a simple means of maximising patients' exposure to natural light/ sunlight could be to ensure that they have regular opportunities to be taken somewhere to experience natural light for a specific period when possible.

It has also been reported that patients admitted to psychiatric hospital for depression may have better clinical outcomes, including shorter and less costly stays, if they occupy sunnier rooms (Benedetti 2001; BMA Science \& Education 2011). This may be important, given the high prevalence of depression in many chronic physical health conditions (National Institute for Health and Clinical Excellence 2011b).

\section{Natural scenes}

The potential benefits that patients may gain from having the opportunity to look at natural scenes or experience nature should be recognised. Empirical findings have suggested that looking at scenes from nature is more conducive to promoting recovery than looking at built scenes (e.g. rooms, buildings and car parks) (BMA Science $\&$ Education 2011). It has been reported that cardiac surgery patients in an ICU where they could look at a picture of a landscape had lower anxiety and stress levels and took less analgesics than a control group with no landscape scene to look at (Ulrich 1991). Likewise, a retrospective analysis of records of surgical patients after cholecystectomy found that those assigned to rooms with windows looking out on a natural scene had shorter postoperative hospital stays, received less negative evaluative comments in nurses' records and were administered fewer potent analgesics than those in rooms with windows facing a brick building wall (Ulrich 1984).

\section{Access to gardens}

The provision of therapeutic garden spaces in hospitals could be important in improving patients' well-being and alleviating the experience of boredom. It has been suggested that hospital gardens reduce stress and improve outcomes not only through the restorative or calming effect of nature, but also through mechanisms such as increased social interaction and the sense of control over stressful clinical settings afforded by being able to escape them for a while (BMA Science $\&$ Education 2011). These findings are of interest considering Binnema's aforementioned (2004) theory that boredom is inherently linked 
to the experience of meaning in life. However, on a practical note, how staff facilitate access to garden spaces is equally important. For instance, will the garden be in enclosed or unenclosed courtyards, lit in the evenings, wheelchair friendly and have sufficient seating? Also, will staff allow or indeed have time to take a patient off the ward?

\section{Noise and hospital layout}

There is evidence that noise can increase stress, blood pressure and heart rate and cause sleep deprivation (BMA Science \& Education 2011). Given the importance of sleep for immune, endocrine and metabolic functions, noisy environments may have a negative effect on well-being and health. The complexity of hospital layouts and poor signage can cause patients to lose their bearings and become stressed, thereby raising cortisol level and lowering functioning of the immune system (Zeisel 2003; BMA Science \& Education 2011). Hospital layouts can also negatively affect staff: for example, in the wards of older hospitals in particular, nurses spend a significant portion of their time walking up and down long corridors (BMA Science \& Education 2011: p. 12). Given this and the many other demands on nurses' time, it might be difficult for them to spend time on activities designed to alleviate boredom and promote patient well-being more holistically.

\section{Challenges for healthcare professionals in the recognition of boredom}

The notion that it is appropriate for clinicians to seek to recognise and alleviate boredom among general hospital in-patients is somewhat at odds with the traditional medical model of healthcare, which focuses on the biological and physiological basis of illness, and in which 'The broader wellbeing of the person experiencing illness [...] can be lost' (BMA Science \& Education 2011: p. 4).

In conceptualising the nuances and wider implications of boredom in general hospitals, it is helpful to consider the difference between 'disease' and 'sickness' in the broader biopsychosocial model. As Dieppe puts it, disease is an 'abnormality of the structure and function of the body' but 'the illness experience and sickness are not always caused by the disease; they depend on psychosocial factors as well' (Dieppe 2005: p. 3). Psychosocial factors such as the experience of boredom therefore interact with the physiological symptoms of disease to lead to a more complex experience of sickness. It is much more straightforward to diagnose and treat 'disease' than to consider the patient's situation in a more holistic manner that encompasses the contribution of myriad psychosocial factors to their well-being and experience of sickness (or wellness).

\section{Clinicians' communication skills}

The ability of clinicians to recognise boredom is partly contingent on their ability to communicate effectively with patients to elicit experiences of boredom and to understand how these experiences may affect patients' broader health status. Communication skills in medicine are often divided into three styles: content skills, process skills and perceptual skills (Box 3). The ability to embody high levels of all three styles is arguably necessary to be an effective practitioner of medicine, but process skills are particularly important for engaging with patients' experiences of boredom and other psychosocial factors. Yet it is precisely these process skills that are underestimated, compared with content skills, in medicine in general, with a tendency for many medics to privilege the 'traditional medical history' over the 'communication model' (Kurtz 2005: p. 34). Patients' psychosocial needs relating to boredom will be missed unless healthcare professionals develop the necessary process communication skills.

In practice, it is difficult for healthcare professionals to engage fully with patients' psychosocial needs and to work to alleviate patient boredom because of other demands on these professionals' time. For example, working with people with cancer, Passik et al (2003) developed the Purposelessness, Understimulation and Boredom (PUB) Scale to detect boredom and differentiate it from depression; but it is difficult to recommend its routine use, given the time pressures on many staff.

BOX 3 Communication skills in clinicians

- Content skills encompass the substance and accuracy of what healthcare professionals communicate to their patients

- Process skills relate to how these professionals communicate content to their patients, i.e. their ability to build a rapport with the patient, structure the interaction appropriately and use verbal and non-verbal skills effectively

- Perceptual skills are concerned with healthcare professionals' problem-solving, diagnostic and interpretive skills and their ability to handle their own and their patients' emotions

(Kurtz 2005) 


\section{Suggestions for further research}

It should be noted that there is a lack of research employing a randomised controlled trial (RCT) design to investigate patient boredom, wellbeing and psychosocial issues in acute hospitals. However, the fact that healthcare systems are so complicated, with myriad potential confounders, makes the RCT design essential for illuminating these issues (Sheldon 2002). Only in RCTs can we disentangle the potential effect of manipulating an independent variable on a dependent variable from the 'noise' of factors attributable to the patients and to other healthcare systems in acute hospitals.

\section{References}

Artaloytia JF, Arango C, Lahti A, et al (2006) Negative signs and symptoms secondary to antipsychotics: a double-blind, randomized trial of a single dose of placebo, haloperidol, and risperidone in healthy volunteers. American Journal of Psychiatry, 163: 488-93.

Bartley E, White J, Janseen $\mathrm{H}$, et al (2012) Exploring the experience of stroke rehabilitation following exposure to an enriched environment [abstract]. International Journal of Stroke, 7 (suppl 1): 44.

Bauld L (2011) The Impact of Smokefree Legislation in England: Evidence Review. Central Office of Information.

Baumann M, Peck S, Collins C, et al (2013) The meaning and value of taking part in a person-centred arts programme to hospital-based stroke patients: findings from a qualitative study. Disability and Rehabilitation, 35: $244-56$.

Beauchemin K, Hays P (1998) Dying in the dark: sunshine, gender and outcomes in myocardial infarction. Journal of the Royal Society of Medicine, 91: 352-4.

Benedetti F, Colombo C, Barbini B, et al (2001) Morning sunlight reduces length of hospitalisations in bipolar depression. Journal of Affective Disorders, 62: 221-3.

Binnema DJ (2004) Interrelations of psychiatric patient experiences of boredom and mental health. Issues in Mental Health Nursing, 25: 833-42.

BMA Science \& Education (2011) The Psychological and Social Needs of Patients. BMA.

Brodrick D, Lewis D, Worth A, et al (2011) One-page patient passport for people with learning disabilities. Nursing Standard, 25: 35-40.

Byrne $\mathrm{P}$ (2000) Stigma of mental illness and ways of diminishing it. Advances in Psychiatric Treatment, 6: 65-72.

Campion J, Checinski K, Nurse J, et al (2008) Smoking by people with mental illness and benefits of smoke-free mental health services. Advances in Psychiatric Treatment, 14: 217-28.

Clissett $P$ (2001) The effect of recreational activities on older people's rehabilitation. Nursing Standard, 15: 39-42.

Conroy RM, Golden J, Jeffares I, et al (2010) Boredom-proneness, Ioneliness, social engagement and depression and their association with cognitive function in older people: a population study. Psychology Health and Medicine, 15: 463-73.

Cumbler E, Mramor W, Hagman J, et al (2011) Purposeful visits for hospitalized elderly patients: program impact on orientation, agitation and mood. Journal of Hospital Medicine, 6: S105-6.

Dieppe P (2005) Research on health and health care. In Handbook of Health Research Methods: Investigation, Measurement and Analysis (eds A Bowling, S Ebrahim): 3-11. Open University Press.

Flaherty AW (2011) Brain illness and creativity: mechanisms and treatment risks. Canadian Journal of Psychiatry, 56: 132-43.

Grady KL, Jalowiec A, White-Williams C (1999) Preoperative psychosocial predictors of hospital length of stay after heart transplantation. Journal of Cardiovascular Nursing, 14: 12-26.
Hardy G, West M (1994) Happy talk. Health Services Journal, 104: 24-6. Higgins R, Hurst K, Wistow G (1999) Nursing acute psychiatric patients: a quantitative and qualitative study. Journal of Advanced Nursing, 25 : 833-42.

Hyland C (1996) Fighting the yawn factor. Health Service Journal, 106 30-1

Inman A, Kirsh KL, Passik SD (2003) A pilot study to examine the relationship between boredom and spirituality in cancer patients. Palliative and Supportive Care, 1: 143-51.

Keefler J, Duder S, Lechman C (2001) Predicting length of stay in an acute care hospital: the role of psychosocial problems. Social Work in Health Care, $33(2): 1-16$

Kurtz S, Silverman J, Draper J (2005) Teaching and Learning Communication Skills in Medicine. Radcliffe.

Lechman R, Duder S (2006) Psychosocial severity, length of stay and the role of social work services. Social Work in Health Care, 43 (4): 1-13.

National Institute for Health and Clinical Excellence (2011a) Service User Experience in Adult Mental Health: Improving the Experience of Care for People Using Adult NHS Mental Health Services (NICE Clinical Guideline 136). NICE.

National Institute for Health and Clinical Excellence (2011b) Depression in Adults Quality Standard (NICE Quality Standard 8). NICE.

Newell SE, Harries P, Ayers S (2012) Boredom proneness in a psychiatric inpatient population. International Journal of Social Psychiatry, 58 : 488-95.

Nisselle A, Hanns S, Green J, et al (2012) Accessing flexible learning opportunities: children's and young people's use of laptops in a paediatric hospital. Technology, Pedagogy and Education, 21: 3-20.

Nolan M, Grant G, Nolan J (1995) Busy doing nothing: activity and interaction levels amongst differing populations of elderly patients. Journal of Advanced Nursing, 22: 528-38.

Park CH, Park TW, Yang JC, et al (2012) No negative symptoms in healthy volunteers after single doses of amisulpride, aripiprazole, and haloperidol: a double-blinded placebo-controlled trial. International Clinical Psychopharmacology, 27: 114-20.

Passik SD, Inman A, Kirsh K, et al (2003) Initial validation of a scale to measure purposelessness, understimulation, and boredom in cancer patients: towards a redefinition of depression in advanced disease. Palliative and Supportive Care, 1: 41-50.

Richter MS, Parkes C, Chaw-Kant J (2007) Listening to the voices of hospitalized high-risk antepartum patient. Journal of Obstetric, Gynecologic and Neonatal Nursing, 36: 313-8.

Routasalo P Tilvis R, Kautiainen $\mathrm{H}$, et al (2009) Effects of psychosocial group rehabilitation on social functioning, loneliness and well-being of Ionely, older people: randomized controlled trial. Journal of Advanced Nursing, 65: 297-305.

Royal College of Psychiatrists (2011) Do the Right Thing: How to Judge a Good Ward. Ten Standards for Adult In-Patient Mental Healthcare (Occasional Paper OP79). Royal College of Psychiatrists.

Sheldon T, Oakley A (2002) Why we need randomised controlled trials. In Clinical Trials (eds L Duley, B Farrell): 13-24. BMJ Books.

Stahl SM (2008) Stahl's Essential Psychopharmacology: Neuroscientific Basis and Practical Applications. Cambridge University Press.

Steele R, Henderson P, Lennon F, et al (2013) Boredom among psychiatric in-patients: does it matter? Advances in Psychiatric Treatment, 19: 259-67.

Taylor G, McNeill A, Girling A, et al (2014) Change in mental health after smoking cessation: systematic review and meta-analysis. BMJ, 348: g1151.

Thomas S, Shattell M, Martin T (2002) What's therapeutic about the therapeutic milieu? Archives of Psychiatric Nursing, 16: 99-107.

Todman M (2003) Boredom and psychotic disorders: cognitive and motivational issues. Psychiatry, 66: 146-67.

Ulrich R (1984) View through a window may influence recovery from surgery. Science, 224: 420-1.

\section{MCO answers \\ 1 e $\quad 2 d \quad 3$ a $\quad 4$ e $5 d$}


Ulrich R (1991) Effects of interior design on wellness: theory and recent scientific research. Journal of Health Care Interior Design, 3: 97-109

Vellone E, Rega ML, Galletti C, et al (2006) Hope and related variables in Italian cancer patients. Cancer Nursing, 29: 356-66.
Wise RA (2004) Dopamine, learning and motivation. Nature Reviews Neuroscience, 5: 483-94.

Zeisel J, Silverstein N, Hyde J, et al (2003) Environmental correlates to behavioural outcomes in Alzheimer's special care units. Gerontologist, 43: 697-711

\section{MCOs}

Select the single best option for each question stem

1 The following can contribute to boredom or falsely present as boredom:

a frontal lobe dementia

b schizophrenia

c Korsakoff dysmnesia

d depression

e all of the above.

2 In research into the interplay between boredom, psychosocial factors and physical health conditions:

a a 2001 study found no difference between severity of psychosocial problems and severity of medical condition as predictors of length of stay

b hope was found to be positively correlated with boredom among cancer patients

c loneliness is not at all common among older people

d social isolation is not at all common among older people

e there is no evidence that elderly patients who lead cognitively unstimulating lives may be at increased risk of cognitive decline.

3 Enforced smoking cessation may contribute to boredom among general hospital inpatients because:

a smoking decreases blood serum levels of MAO-B, which causes dopamine levels to increase; stopping smoking will therefore decrease dopamine, and this may contribute to boredom because dopamine is important for motivation, pleasure and reward

b smoking increases blood serum levels of MAO-B, which causes dopamine levels to increase; stopping smoking will therefore increase dopamine, and this may contribute to boredom because dopamine is important for motivation, pleasure and reward

c smoking increases blood serum levels of MAO-B, which causes dopamine levels to decrease; stopping smoking will therefore decrease dopamine, but this will have no effect on boredom because dopamine is not important for motivation, pleasure and reward

d smoking decreases blood serum levels of MAO-B, which causes dopamine levels to decrease; stopping smoking will therefore decrease dopamine, but this will have no effect on boredom because dopamine is not important for motivation, pleasure and reward

e smoking decreases blood serum levels of MAO-B, which causes dopamine to increase; we cannot say whether an increase in dopamine may contribute to boredom because we do not know whether dopamine is important for motivation, pleasure and reward.

4 Nature and hospital gardens may play a part in improving hospital patients' well-being because:

a hospital gardens can promote social interaction

b looking at built scenes may be more stressful to patients than looking at nature scenes c hospital gardens can give patients a sense of control in the midst of stressful clinical settings

$\mathrm{d}$ looking at built scenes is less conductive to patients' recovery than viewing natural scenes

e all of the above hold true.

5 Regarding the interplay between in-patients' psychosocial needs and boredom:

a engaging with patients' psychosocial needs and thereby addressing their boredom is consistent with the traditional medical mode

b engaging with patients' psychosocial needs and thereby addressing their boredom requires content communication skills rather than process communication skills

c engaging with patients' psychosocial needs and thereby addressing their boredom entails a focus on 'disease' rather than 'sickness'

d engaging with patients' psychosocial needs and thereby addressing their boredom necessitates a focus on 'sickness' rather than 'disease'

e engaging with patients' psychosocial needs and thereby addressing their boredom requires doctors to structure their patient interactions by using the 'traditional medical history' rather than the 'communication model'. 